\title{
Traffic Generation of IEC 61850 Sampled Values
}

\author{
Jakub W. Konka*, Colin M. Arthur ${ }^{\dagger}$, Francisco J. Garcia ${ }^{\dagger}$ and Robert C. Atkinson* \\ *Dept. of Electronic and Electrical Engineering \\ University of Strathclyde \\ Glasgow G1 1XW \\ Email: jakub.konka@strath.ac.uk,r.atkinson@eee.strath.ac.uk \\ †Measurement Research Laboratory \\ Agilent Technologies \\ Edinburgh EH12 9DJ \\ Email: \{colin_arthur, frankie_garcia $\} @$ agilent.com
}

\begin{abstract}
The work presented in this paper is targeted at the first phase of the test and measurements product life cycle, namely standardisation. During this initial phase of any product, the emphasis is on the development of standards that support new technologies while leaving the scope of implementations as open as possible. To allow the engineer to freely create and invent tools that can quickly help him simulate or emulate his ideas are paramount. Within this scope, a traffic generation system has been developed for IEC 61850 Sampled Values which will help in the evaluation of the data models, data acquisition, data fusion, data integration and data distribution between the various devices and components that use this complex set of evolving standards in Smart Grid systems.
\end{abstract}

\section{INTRODUCTION}

The utility industry across the globe has been investigating possible ways of addressing grand challenges such as generation diversification, more effective electricity demand response, more efficient energy conservation, and reduction of the industry's overall carbon footprint [1]. As one viable solution the concept of a 'Smart Grid' has been proposed. A Smart Grid can be defined as a unified, fully interoperable, communications-enabled electrical system which aims at revolutionising traditional power systems through the introduction of condition monitoring and intelligence.

Many of the existing standards are being updated and new standards are being developed incrementally in order to ensure a timely and smooth transition from contemporary electrical transmission systems to Smart Grids [2]. One such example is the international standard IEC 61850. Originally developed to define communications within electrical substations, it has recently been extended to cover Smart Grid relevant aspects, such as communications between substations, hydro power plants and distributed energy resources (DERs) [3].

IEC 61850 standardises the way devices at the substation level (and beyond) should communicate with each other to achieve full interoperability. This is crucial for Smart Grids as in such a complex system there is no room for possible communication breakdowns between equipment provided by different vendors. Therefore, thorough understanding of the standard, and existence of realistic and accurate simulation models of the protocols specified therein is a necessity.
Furthermore, as intelligent energy management evolves, like with the Internet and Cyberspace, tussle will exist due to the many different stakeholders [4]. In the case of intelligent energy management the stake holders will include transmission system operators, distribution network operators, equipment manufacturers, regulators, ICT service providers, governments, etc. Where tussle exists, it becomes imperative to ensure that rigorous validation, verification and conformance testing processes are put in place to ensure interoperability and facilitate open competition in a thriving market place.

The test and measurement product life cycle can be broadly categorised into standardisation, certification, interoperability (IOT) field trials, monitoring and optimisation. For a test and measurement organisation, the goal is to deliver a set of test and measurement tools that can speed up the process of any product moving through this cycle to get it into the market place as fast as possible. The tools developed will help any organisation take their product from research and development, through validation, manufacturing, deployment and future evolution.

The work presented in this paper is targeted at the first phase of the test and measurements product life cycle, namely standardisation. During this initial phase of any product, the emphasis is on the development of standards that support new technologies while leaving the scope of implementations as open as possible. To allow the engineer to freely create and invent tools that can quickly help him simulate or emulate his ideas are paramount. Within this scope, a traffic generation system has been developed for IEC 61850 Sampled Values which will help in the evaluation of the data models, data acquisition, data fusion, data integration and data distribution between the various devices and components that use this complex set of evolving standards in Smart Grid systems.

The paper is structured as follows. In Section II a brief overview of IEC 61850 standard is given. Section III summarises simulation modelling research of the topic conducted by other researchers. Section IV describes building blocks of the Sampled Values traffic generator, and Section V presents the steps taken and results of the validation process. Finally, Section VI discusses future work and Section VII draws conclusions. 


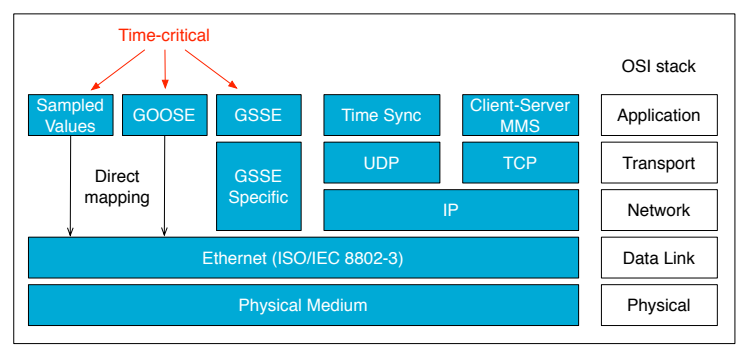

Fig. 1. Communication services defined in IEC 61850

\section{OVERVIEW OF IEC 61850}

The first edition of the IEC 61850 standard comprises 12 parts in total. Each part describes different aspects of the communications taking place within a substation automation system. For example, part 7 presents the logical view of the communication system, i.e., logical models of devices, abstract communication service interface (ACSI), etc., while parts 8 and 9 specify how these logical concepts ought to be mapped into a specific communications infrastructure, i.e., protocol stack.

Throughout this investigation part 9-2 of the standard was scrutinised in-depth since it describes how Sampled Values protocol should be designed and mapped to Ethernet (ISO/IEC 8802-3). Part 9-1 was also analysed, however, in less detail since it is likely to be withdrawn from the future releases of the standard [5].

IEC 61850 defines 5 types of communication services as shown in Fig. 1. The first three types are time-critical and are used in protection and control schemes of the substation. They include Sampled Values (SV) and Generic Object Oriented Substation Event (GOOSE) protocols which are mapped directly to Data Link layer for reduced protocol overhead and hence increased performance; and Generic Substation State Event (GSSE) protocol which features its own custom protocol mapping. The remaining two types of services, i.e., Time Sync and Client-Server Manufacturing Message Specification (MMS), deal with time synchronisation and management of the substation devices respectively.

In order to better understand how these services are used within a substation consider the simplified protection \& control system shown in Fig. 2. A Merging Unit (MU) accepts multiple analogue current and voltage samples coming from current and voltage transformers which are connected directly to the power line. It then acts as an analogue-to-digital-converter converting the samples into digital format and encapsulating them along with meta-data to put these measurements in context as SV packets, and sends them over the switched Ethernet network to Protection \& Control (P\&C) units [6]. The $\mathrm{P} \& \mathrm{C}$ extracts the samples, and examines them to detect evidence of a fault in the protected zone of the network. If the outcome of the examination is positive, it issues a Trip message encapsulated as GOOSE/GSSE packet to the Circuit Breaker (CB) unit which then trips the appropriate switchgear and isolates the fault [7]. Afterwards, CB advises

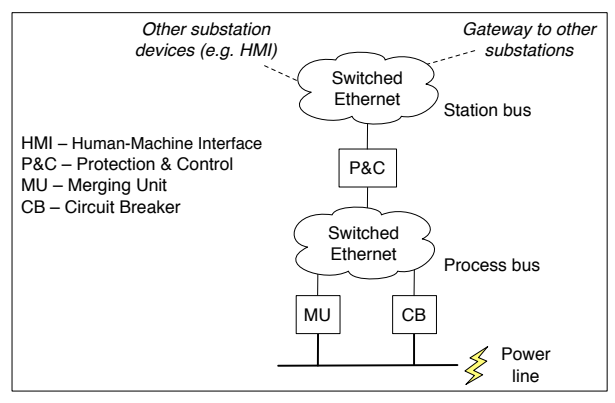

Fig. 2. Simplified protection \& control system used within a substation

other monitoring units of the action by issuing GOOSE/GSSE packet. The exchange of time-critical messages described above is based on the publisher/subscriber messaging model in which one or more subscribers subscribe to the publisher stating that they want to be notified of a particular event (in this case, to act as sinks for SV or GOOSE/GSSE packets).

Time Sync service which was not mentioned in the example above is substation-wide in scope and is used to distribute common timing information across substation devices which require such information. For instance, MUs in order to correctly handle processing of current and voltage samples need to be provided with timing information. The two most popular methods for time synchronisation in the industry include Global Positioning System (GPS) and the more recent IEEE 1588 Precision Time Protocol (PTP).

Client-Server MMS, similarly to Time Sync service, is substation-wide in scope and is used to manage substation devices attached to both process and station buses. In operation, it is quite similar to Simple Network Management Protocol (SNMP), and is also based on the client/server messaging model in which the client polls the server for specific data.

The criticality of rigorous assessment and appraisal of interoperability is inherent to the widespread adoption of this standard within an industry that is risk averse and safety critical. A facility to validate protection and automation functionality within an increasingly integrated ICT system supporting the Smart Grid is essential to its realisation. This work supports this ambition.

\section{LITERATURE OVERVIEW}

Several research investigations looked into the topic of simulation modelling of communication services defined in the IEC 61850 standard. Sidhu and Yin produced two publications [8], [9] showing how the OPNET Modeler network simulator can be used to model the substation communication network and analyse the network's dynamic performance (i.e., packet delay characteristics of time-critical services). Their model of SV traffic generator mapped packets directly onto Ethernet and, in their latter publication, included handling of IEEE 802.1Q VLAN tags. Moreover, in [9], the model additionally appended SV-specific header, application service data units (ASDUs), and application protocol data unit (APDU) according to IEC 61850-9-1. 


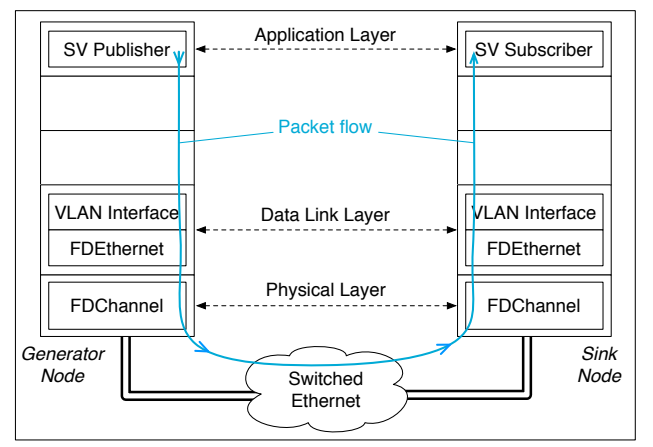

Fig. 3. OSI-7 reference model of the SV traffic generator

Kanabar et al. [10] presented results of modelling communication networks for distributed automation systems with DERs in the OPNET Modeler. In their investigation, SV protocol was mapped directly onto Ethernet layer, and included VLAN tags. However, no information was provided whether SV-specific header, ASDUs and APDU were also appended. Similarly, Ali and Thomas [11] did not specify whether any SV-specific fields were attached to the packet while modelling various communication scenarios within a substation in the OPNET Modeler.

Finally, Liang and Campbell [12] concentrated on implementing and simulating ACSI in J-Sim open-source simulator. Their goal was to explore security aspects of the standard and in particular of IEC 61850-7.

\section{DESCRIPTION OF THE TRAFFiC Generator}

The model of SV traffic generator developed during this investigation is based on part 9-2 of the IEC 61850 standard [13], [14], and on IEC 61850-9-2 LE implementation guidelines [15]. It was implemented in NS3 open-source network simulator, version 3.8 [16]. NS3 was chosen out of many other available simulators for two reasons: 1) it is opensource, hence it is possible to alter the core of the simulator if necessary; and 2) it features uniform coding structure, i.e., both simulation scripts and its core are written in $\mathrm{C}++$ programming language, hence there is no need to create language bindings as is the case in NS2 for example (OTcl/C++ bindings).

Fig. 3 depicts a conceptual model of the traffic generator in the spirit of OSI-7 reference model. The generator node is responsible for generating packets carrying SV protocol (or simply SV packets) while the sink node acts as their addressee. Both nodes implement 3 out of 7 OSI layers as required by [14]: application, data link, and physical layer. The functionality of the application layer is implemented by $S V$ Publisher application in the generator node, and by $S V$ Subscriber application in the sink node. The functionality of the data link layer is split into two parts: VLAN Interface and FDEthernet sublayers; while the functionality of the physical layer is implemented by FDChannel. VLAN Interface and FDEthernet sublayers together with FDChannel model the behaviour of full-duplex Ethernet with IEEE 802.1Q support,

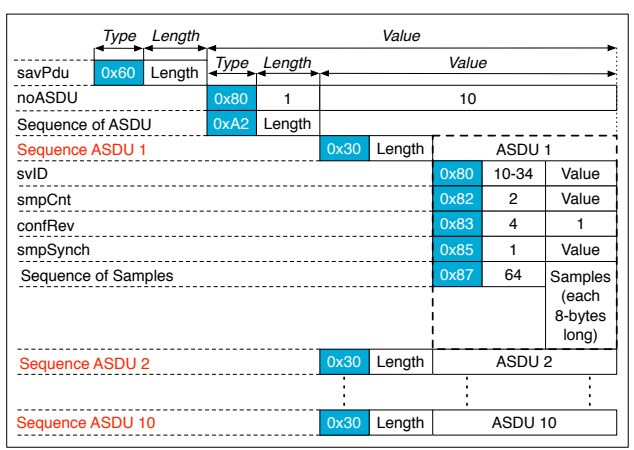

Fig. 4. Structure of modelled SV APDU/ASDU (ASN.1/BER TLV triplets)

i.e., payload coming from upper layers is encapsulated inside the Ethernet-II (EtherType) type framing with VLAN tagging.

All parts of the traffic generator were fully designed and engineered in the simulator in order to gain first-hand experience in working with the standard. Moreover, since the main aim was to create a realistic traffic generator, as packets go up/down the protocol stack appropriate headers and trailers are appended/removed on a layer basis similarly to show how it occurs in a real communication scenario.

\section{A. SV Publisher/Subscriber Application}

Every SV packet is created by SV Publisher application, and upon creation consists of a series of SV ASDUs encapsulated inside the SV APDU. The byte structure of SV APDU and SV ASDU implemented in the model is based on the Abstract Syntax Notation One (ASN.1) and Basic Encoding Rules (BER) Type-Length-Value (TLV) triplets specified in [14]. The structure is shown in Fig. 4. At present, the model of SV APDU/ASDU does not include any of the optional TLV triplets, i.e., datSet, refrTm, smpRate and smpMod. Moreover, the majority of the implemented triplets have their Value fields set to 0 or an arbitrary value to simply preserve the structure of SV APDU/ASDU rather than carry any meaningful information. Future work will involve adding optional triplets, and setting Value fields to something more meaningful as the SV traffic generator will be improved, and models of other protocols such as GOOSE/GSSE will be added.

SV-specific header is appended to SV APDU before it is sent down the protocol stack to lower layers. The header is 8 bytes long and comprises 4 fields: APPID, Length, Resrv1, and Resrv2 (see Fig. 5, topmost packet structure). The APPID field stands for Application Identifier [14], and is used to select those Ethernet frames which contain SV APDU, and to distinguish between GOOSE/GSSE and SV protocols. APPID is always 2 bytes long and in case of SV protocol has the two most significant bits (MSBs) set to 01, while the remaining 14 bits are the actual ID used within the substation communication network. Therefore, the values of APPID range from $0 \times 4000$ to $0 x 7 \mathrm{FFF}$. By default, it should be set to $0 \times 4000$.

The Length field is also 2 bytes long, and indicates the length of the packet including the SV-specific header, i.e., if 


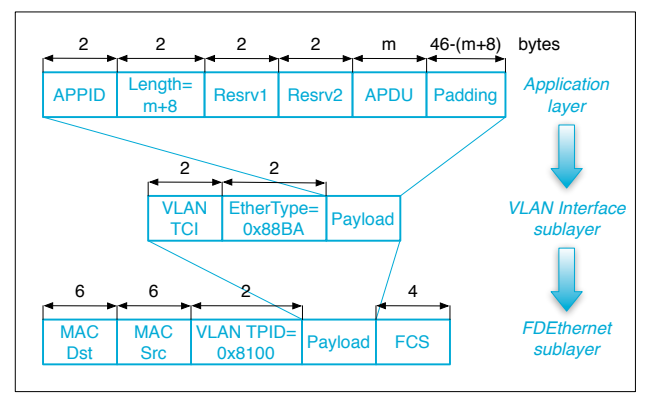

Fig. 5. Encapsulation of SV APDU as it goes down the protocol stack

the length of the APDU is $m$ bytes and since the length of the SV-specific header is 8 bytes, Length field shall be set to $m+8$.

The Resrv1 field stands for Reserved 1. It is 2 bytes long, and features a set of blocks of bits each serving different function. The MSB, if set to 1, indicates that this SV packet has been issued by a test device. The following 3 bits are reserved for future standardised application, and by default, are set to 0 . The remaining 12 bits are reserved for security purposes, and by default, should also be set to $0 x 000$. The Resrv2 field stands for Reserved 2. It is 2 bytes long, and is used to denote additional security parameters carried by this SV packet. By default, it should be set to 0x0000.

In our model, APPID and Resrv2 fields assume their default values, that is APPID is set to $0 \times 4000$ and Resrv2 to $0 x 0000$. Resrv1 field is set to $0 \times 8000$ to indicate that packets were generated by a test device. Length field is updated automatically based on the actual length of SV APDU. If the total length of the packet (SV APDU + SV-specific header) is smaller than 46 bytes, SV Publisher application will pad it with zeros in order to ensure that it conforms with the requirements of Ethernet, i.e., the minimum length of the payload encapsulated inside the Ethernet frame must not be smaller than 46 bytes.

The SV Subscriber application on the other hand acts as a simple sink for SV packets. It basically removes SVspecific header and padding (if any) from a packet which was forwarded up to the application by VLAN Interface sublayer.

\section{B. VLAN Interface Sublayer}

A VLAN Interface sublayer was designed to imitate the behaviour of an IEEE 802.1Q VLAN-aware end station/device, i.e., a network node which is capable of explicitly VLAN tagging the frame before it is ever sent out [17]. It is clearly stated in [14] that frames carrying SV APDU have to be VLAN tagged explicitly. The main task of this sublayer is to append/remove VLAN Tag Control Information (TCI) and EtherType field to/from the packet received from upper/lower layer (see Fig. 5, middle packet structure). The VLAN TCI comprises three fields [17]: Priority, Canonical format indicator (CFI), and VLAN ID.

The Priority field is 3 bits long, and is used to denote the priority which the frame should be treated with when travelling through priority-aware switched Ethernet network. Its value can range from 0 to 7 according to IEEE $802.1 \mathrm{p}$. There are no strict rules as to how those values should be distributed among different types of traffic but, by default, for SV-type payload, priority should be set to 4 [14].

The CFI field is one bit long, and in general, refers to the bit ordering format of the bytes within a frame. Its precise meaning however depends on the underlying technology (Ethernet, Token Ring, etc.). In Ethernet networks, frames are normally sent with CFI bit set to 0 .

The VLAN ID field is 12 bits long, and is used to explicitly identify frame's VLAN association. For SV-type payload, if no VLAN segments were configured, it should be set to 0x000 [14]. Otherwise, since SV protocol needs to have potentially its own bandwidth allocation, it should be set to a value different from the one used by GOOSE/GSSE protocols.

The value of each of the VLAN TCI composite fields is determined at the application layer, hence in this case by SV Publisher application, and is passed to VLAN Interface sublayer together with the pointer to SV packet and the value of EtherType field. The model assumes the following default values: Priority set to 4 , CFI to 0 , and VLAN ID to $0 \times 000$. The EtherType field which uniquely identifies the payload/protocol carried within the Ethernet frame is set to SV Protocol ID, that is $0 \mathrm{x} 88 \mathrm{BA}$

When the packet is received from the lower layer, the VLAN Interface sublayer removes VLAN TCI and EtherType field. It then inspects the removed EtherType field and compares it with the SV Protocol ID. If the two match, the packet is forwarded up the protocol stack to SV Subscriber application. Otherwise, the packet is dropped at this sublayer. This mechanism was developed to ensure that only VLAN tagged frames carrying SV-type payload are forwarded up to SV Subscriber application.

\section{FDEthernet Sublayer}

The FDEthernet sublayer was designed to imitate the behaviour of full-duplex Ethernet network interface card. Therefore, its main functions include: enqueueing/dequeueing frames for transmission over FDChannel; appending/removing destination and source MAC address fields, and VLAN Tag Protocol Identifier (TPID) field to/from the frame going up/down the protocol stack (see Fig. 5, lowermost packet structure); and calculating and appending/removing the Frame Check Sequence (FCS).

The value of VLAN TPID (i.e., VLAN Protocol ID, 0x8100) is provided to FDEthernet sublayer by VLAN Interface together with the pointer to payload. When packet is received from lower layer, i.e., FDChannel, and passes checksum control, FDEthernet sublayer forwards the frame up the protocol stack only if the third field of the EthernetII header (i.e., EtherType field if no VLAN tagging is employed) matches the VLAN Protocol ID. Otherwise, the packet is discarded at this sublayer. This mechanism ensures that only VLAN tagged frames are forwarded to VLAN Interface sublayer. 
Moreover, since frames carrying SV-type payload are VLAN tagged, the Ethernet maximum transmission unit (MTU) needs to be increased from 1,518 to 1,522 bytes according to IEEE 802.3ac supplement [17]. MTU is used to determine maximum frame length (Ethernet-II header inclusive) at the Ethernet layer which if exceeded causes frames to be fragmented. In our model, MTU is executed by FDEthernet sublayer.

\section{FDChannel}

FDChannel acts as a physical transmission medium, and can be configured to behave like any type of full-duplex physical medium, e.g. twisted pair or fibre link.

\section{Model Validation}

In order to validate the model of SV traffic generator, a simple simulation scenario was created. Packet traces gathered from the simulation were saved in PCAP file format which is natively supported by NS3 [16], and can be analysed in Wireshark network protocol analyser [18]. If the structure of generated SV packets is dissected by Wireshark correctly, we can conclude that the traffic generator behaves as required.

\section{A. Simulation Scenario}

The topology of the simulated network consisted of 4 nodes: 2 SV Publisher end devices (node0 and node1) were generating SV packets and sending them to SV Subscriber end device (node2) through a priority-aware L2 Ethernet switch. All links were modelled as full-duplex with capacity of $100 \mathrm{Mbps}$ and propagation delay of $1 \mathrm{~ms}$.

Each generated SV packet was 997 bytes long including all the headers, i.e., SV-specific header, VLAN tags and EthernetII header (1001 bytes including FCS). Each SV APDU was 971 bytes long and comprised 10 SV ASDUs, each 96 bytes long. Moreover, each SV ASDU comprised: buffer worth of 8 sampled values (64 bytes long) padded with zeros; Value field of svID TLV triplet set to "NS3Generated" (13 bytes long including null character); Value fields of confRev and smpSynch TLV triplets set to 1; and all other Value fields of the remaining (non-optional) TLV triplets set to 0. Furthermore, node0 generated SV packets with the total bit rate of $80 \mathrm{Mbps}$, while nodel with the total bit rate of $70 \mathrm{Mbps}$.

\section{B. Simulation Results}

Packet traces were gathered at the SV Subscriber node (node2). Furthermore, in all of the traces, SV nodes assume the following MAC addresses: 00:00:00:00:00:01 for node0, 00:00:00:00:00:03 for node1, and 00:00:00:00:00:05 for node2.

Traces were analysed in the latest stable release of Wireshark (at the time of writing; version 1.4.0) [18], on Ubuntu Linux 10.04 .

Fig. 6a shows the result of dissecting SV packets by Wireshark. The length of the SV packet including all the headers, i.e., SV-specific header, VLAN tags and EthernetII header, is displayed to be 997 bytes as desired. Moreover,
SV-specific header has its fields set in accordance with the assumptions of the modelling process: APPID is set to 0x4000, Resrv1 to 0x8000 and Resrv2 to 0x0000. Length field, which is supposed to indicate the actual length of SV APDU including the length of SV-specific header, is set to 979. It agrees with the assumptions stated earlier and is also proven to be the case by Wireshark - the bottom of Fig. 6a shows that Wireshark interpreted the length of SV APDU including SV-specific header to be 979 bytes as well. SV APDU is shown to consist of $10 \mathrm{SV}$ ASDUs as desired. Furthermore, Value fields of TLV triplets in each SV ASDU also correspond to the assumed values, for example $s v I D$ is set to "NS3Generated" as assumed in Section V-A.

Finally, Fig. 6b depicts the contents of the buffer which carries sampled values of current and voltage. Although in our model the buffer is padded with zeros throughout, its structure is intact and can be populated with real values in the future. All in all, this proves that our SV traffic generator successfully models the structure of SV packets accurately.

\section{FUTURE WORK}

There are a number of potentially fruitful research issues worthy of further investigation. Firstly, the model of SV traffic generator should be improved by feeding SV APDU/ASDU with realistic data. The more realistic the traffic generator, the more accurate the output from the simulator and hence, the greater the insight into different issues of communications within the substation (and beyond). Traffic with realistic data allows to better scale the network still in the simulation modelling stage; thus, hastening the process of installing the communications network as well as making it more reliable from the very beginning.

Secondly, the model of SV traffic generator described in this paper could be used as a building block for GOOSE and GSSE traffic generators. Implementation of these two protocols is the next step in understanding the IEC 61850 standard thoroughly and creating a realistic simulator of the substation communication network based on this standard.

Lastly, models of Time Sync and Client/Server MMS services should be added and tied up with models of GOOSE/GSSE and SV protocols. This would pave the way for more Smart Grid relevant simulation research such as modelling of communications between substations.

\section{CONCLUSIONS}

Smart Grids are a major leap forward in the functionality of electrical systems. As such, this new emerging technology requires advanced and "bullet-proof" communications on all levels of abstraction. The international standard IEC 61850 is the most likely candidate for standardisation of communications on substation level and beyond. Therefore, its thorough understanding and existence of accurate and realistic models of the protocols and communication services described therein is a necessity.

In this paper, an accurate and realistic model of Sampled Values traffic generator was described. Packet traces generated 


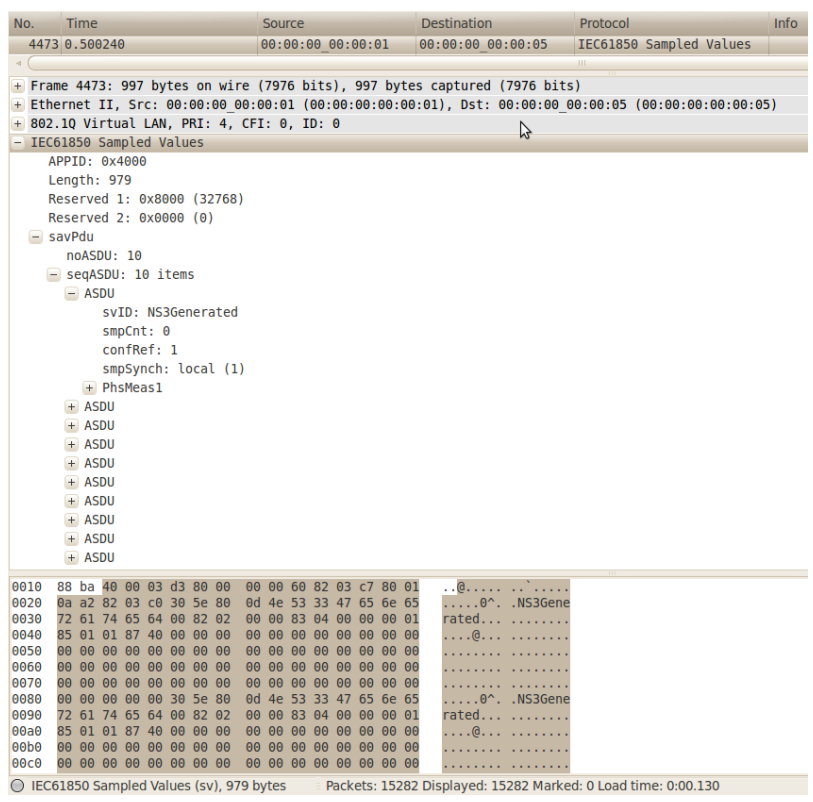

(a) SV-specific header and SV APDU/ASDU

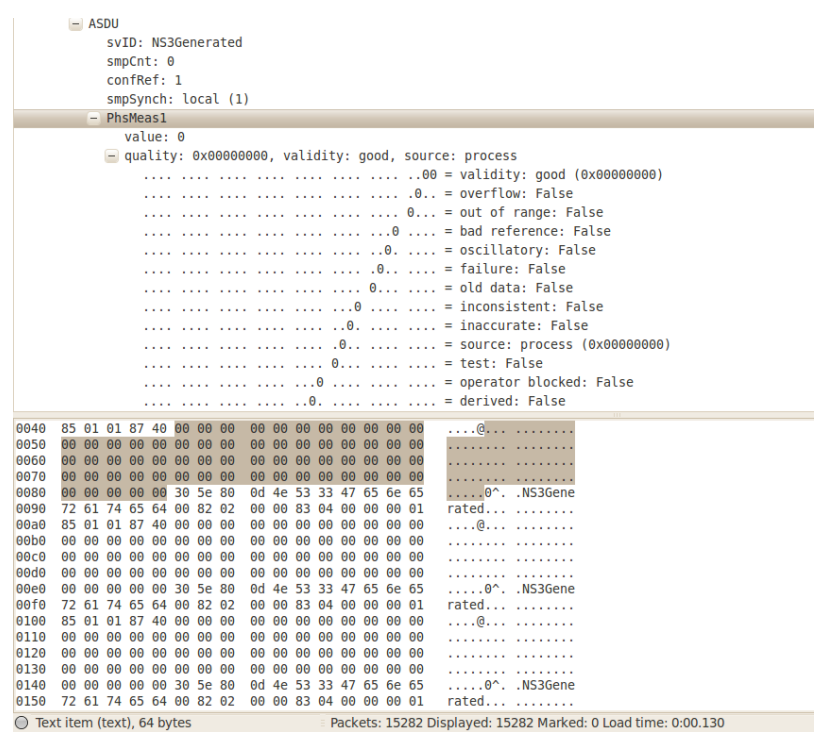

(b) Buffer containing sampled values

Fig. 6. Structure of SV packets generated during the simulation dissected by Wireshark

using the model were shown to be realistic as they included the full byte structure of Sampled Values APDU and ASDU. Moreover, they were also correct since Wireshark network protocol analyser dissected them without any glitches whatsoever.

The model can be used to assess all critical to safety first utility applications such as: the interoperability with realistic traffic levels; the assessment of bandwidth and potential erroneous operation; resilience to interference/noise; etc. Furthermore, the development of the model from first principles during this investigation proved to be very useful and gave great insight into how communications is managed in the IEC 61850-enabled substation.

\section{ACKNOWLEDGMENTS}

The authors would like to thank Peter Cain from Agilent Technologies for providing access to an up-to-date report from NIST proceedings on the development and standardisation of Smart Grids, and Paul Crolla, Dr. James Irvine and Professor Graeme Burt from University of Strathclyde for their valuable comments on the contents of this paper.

\section{REFERENCES}

[1] H. Farhangi, "The Path of the Smart Grid," in IEEE Power and Energy Magazine, vol. 8, pp. 18-28, Jan.-Feb. 2010.

[2] NIST, "NIST Framework and Roadmap for Smart Grid Interoperability Standards," Release 1.0. NIST Special Publication 1108. Office of the National Coordinator for Smart Grid Interoperability, January 2010.

[3] International Standard "IEC 61850: Communication networks and systems for power utility automation." IEC, 2002-10.

[4] D. D. Clark and J. Wroclawski, "Tussle in Cyberspace: Defining Tomorrow's Internet," in SIGCOMM'02, 19-23 August 2002, Pittsburgh, Pennsylvania, USA.

[5] K. Schwarz, "What is Edition 1 and Edition 2 of IEC 61850?" News on IEC 61850 and Related Standards, Mar. 3, 2010. [Online]. Available: http://iec61850-news.blogspot.com/2010/03/ what-is-edition-1-and-edition-2-of-iec.html. [Accessed: Aug. 23, 2010].
[6] M. C. Janssen and A. Apostolov, "IEC 61850 Impact on Substation Design," in Transmission and Distribution Conference and Exposition 2008. T\&D. IEEE PES, pp. 1-7, April 2008.

[7] H. Ito and K. Ohashi, "High Performance IEC 61850 GOOSE and Protection Relay Testing," in PAC World Magazine, Winter 2008 Issue. [Online]. Available: http://www.pacw.org/no-cache/issue/winter_ 2008 issue/protection_goose/high_performance_iec_61850_goose and_protection_relay_testing.html. [Accessed: Aug. 23, 2010].

[8] T. S. Sidhu and Y. Yin, "IED Modelling for IEC61850 Based Substation Automation System Performance Simulation," in Power Engineering Society General Meeting, 2006. IEEE, pp. 1-7, 18-22 June 2006.

[9] T. S. Sidhu and Y. Yin, "Modelling and Simulation for Performance Evaluation of IEC61850-Based Substation Communication Systems," in IEEE Transactions on Power Delivery, vol. 22, pp. 1482-1489, July 2007.

[10] P. M. Kanabar, M. G. Kanabar, W. El-Khattam, T. S. Sidhu and A. Shami, "Evaluation of Communication Technologies for IEC 61850 based Distribution Automation System with Distributed Energy Resources,' in Power \& Energy Society General Meeting, 2009. PES '09. IEEE, pp. 1-8, 26-30 July 2009.

[11] I. Ali and M. S. Thomas, "Substation Communication Networks Architecture," in Power System Technology and IEEE Power India Conference, 2008. POWERCON 2008. Joint International Conference on, pp. 1-8, 12-15 Oct. 2008.

[12] Y. Liang and R. H. Campbell, "Understanding and Simulating the IEC 61850 Standard," 2008. [Online]. Available: https://www.ideals.illinois. edu/handle/2142/11457. [Accessed: Aug. 23, 2010].

[13] International Standard "IEC 61850-9-2: Communication networks and systems for power utility automation - Part 9-2: Specific Communication Service Mapping (SCSM) - Sampled values over ISO/IEC 8802-3," 1st ed. IEC, 2004.

[14] International Standard "IEC 61850-9-2: Communication networks and systems for power utility automation - Part 9-2: Specific Communication Service Mapping (SCSM) - Sampled values over ISO/IEC 8802-3," 2nd ed. Draft for public comment. IEC, 2009.

[15] UCA International Users Group, "IEC 61850-9-2 LE: Implementation Guideline for Digital Interface to Instrument Transformers Using IEC 61850-9-2." UCA, 2004.

[16] NS3 Open-Source Network Simulator, version 3.8. [Online]. Available: http://www.nsnam.org. [Accessed: Aug. 23, 2010].

[17] R. Seifert and J. Edwards, The All-New Switch Book: The Complete Guide to LAN Switching Technology, 2nd ed. Indianapolis, IN: Wiley Publishing, 2008.

[18] Wireshark Network Protocol Analyzer, version 1.4.0. [Online]. Available: http://www.wireshark.org. [Accessed: Aug. 30, 2010]. 